Journal of the Intensive Care Society 2015, Vol. 16(3) 244-246

\title{
Changing patterns of in-hospital deaths following implementation of damage control resuscitation practices in US forward military treatment facilities
}
(C) The Intensive Care Society 2015 Reprints and permissions: sagepub.co.uk/ journalsPermissions.nav DOI: $10.1177 / 1751143715591766$ jics.sagepub.com ๑SAGE

Introduction of Damage Control Resuscitation Practices was associated with an increase in Injury Severity Score of patients that died.

Level of Evidence: 3 (Evidence from non-experimental descriptive studies, such as comparative studies, correlation studies, and case-control studies).

Appraised by: Charles Handford, Tomasz Torlinski

Authors: Nicholas R Langan, MD; Matthew Eckert, MD; Matthew J Martin, MD.

Citation: Langan NR, Eckert $M$ and Martin MJ. Changing patterns of in-hospital deaths following implementation of damage control resuscitation practices in US forward military treatment facilities. JAMA Surg 2014; 149: 904-912.

Introduction of Damage Control Resuscitation Practices was associated with an increase in Injury Severity Score of patients that died.

Level of Evidence: 3 (Evidence from nonexperimental descriptive studies, such as comparative studies, correlation studies, and case-control studies).

Three-part clinical question

Patients: Patients treated at a United States (US) Military Treatment Facility (MTF) and who subsequently died in a US MTF.

Intervention: Damage control resuscitation (DCR). In this article, the authors describe DCR in the following way: 'The basic principles of DCR include the early administration of blood products in a balanced ratio, aggressive correction of coagulopathy, and the minimization of crystalloid fluids. ${ }^{1,2}$

Outcome: Primary outcome consisted of severity of injury (via Injury Severity Score and other well recognized markers). Secondary outcomes were: packed red blood cell to fresh frozen plasma administration ratio (PRBC:FFP), total volume of all blood products and fluid administrated, timing/location of death and area of injury.

Study design: Retrospective analysis of prospectively collected data, via the Joint Theater Trauma Registry (JTTR), split into two cohorts (pre 2006 and 20062011). A split at pre/post 2006 was chosen as in the latter half of 2005 DCR policies and DCR-based massive transfusion clinical guidelines were implemented. As a result of this, patients treated after this point should have been resuscitated according to the principles of DCR.

The study patients

Eligible: Soldiers (patients) treated in a US MTF who died in hospital during Operation Iraqi Freedom (OIF) and Operation Enduring Freedom (OEF).

Included: 2565 patients met the above eligibility criteria: 902 patients before January 2006, 1663 between 2006 and 2011.

Exclusion criteria: All patients in the registry who survived their injury. Patients who had no recorded vital signs (blood pressure and heart rate) on arrival as they were assumed to be dead on arrival.

Control group: $(\mathrm{n}=902)$. Received fluid resuscitation prior to wide scale introduction of DCR (prior to January 2006).

Intervention group: $(n=1663)$. Received fluid resuscitation following the wide scale implementation of DCR (2006-2011).

Author's hypothesis: Independent survival benefit of DCR should also be reflected by corresponding change in the epidemiology of in-hospital trauma deaths.

\section{Results (Table 1)}

After the introduction of DCR, the mean ISS in nonsurvivors increased from 22.5 to 26.7 . The proportion of severe injury increased from 63.5 to $79.7 \%$ $(\mathrm{p}<0.05)$. As a comparison to this apparent trend all those on the JTTR including survivors were analyzed. This was to ensure that the apparent trend in 
Table I. Deaths and ISS.

\begin{tabular}{llll}
\hline & Pre-DCR & Post-DCR & P \\
\hline $\begin{array}{l}\text { Data } \\
\quad \text { Years }\end{array}$ & $2001-2005$ & $\begin{array}{l}2006-200 \mid \\
1663\end{array}$ \\
$\quad \begin{array}{l}\text { Patient, no. } \\
902\end{array}$ & & \\
$\begin{array}{l}\text { Deaths, \% } \\
\text { Early (0-24h) }\end{array}$ & 77 & 80 & 0.02 \\
Intermediate & 13 & 13 & .95 \\
$\quad$ I-7 d) & & & \\
Late (>7 d) & 13 & 6 & $<0.01$ \\
ISS & & & \\
Mean ISS & 22.5 & 26.7 & 0.03 \\
ISS > I5\% & 63.5 & 79.7 & $<0.01$ \\
\hline
\end{tabular}

ISS: Injury Severity Score; DCR: damage control resuscitation.

non-survivors did not represent an increase in ISS in all those injured, including survivors. No difference when survivors were included in the analysis was found between the two time periods. When assessing area of injury there was significantly $(\mathrm{p}<0.01)$ more head injuries but no significant change to other body regions.

When looking at timing of deaths it was noticed that post 2006, there was a significant increase in the proportion of early deaths but a reduction in the proportion of late deaths.

Post 2006, PRBC and FFP usage has increased $(\mathrm{p}=0.01)$. Alongside this the $\mathrm{PRBC} / \mathrm{FFP}$ ratio also changed from 2.6:1 to $1.4: 1 \quad(p<0.01)$. Crystalloid usage was significantly reduced (from $6.1 \mathrm{~L}$ to $3.2 \mathrm{~L}$ ). In terms of all other fluids and blood products there was no significant change between the groups.

\section{EBM questions:}

1. Do the methods allow accurate testing of the hypothesis? No. The methods enable associations to be drawn, however, due to lack of controls and multiple potential confounders it is hard to truly test the given hypothesis that DCR gives rise to an independent survival benefit.

2. Do the statistical tests correctly test the results to the differentiation of statistically significant results? Yes. T-test/Mann-Whitney $U$ test for continuous data. $\chi^{2}$ for categorical data.

3. Are the conclusions valid in the light of the results? Partially. The three main conclusions are as follows: (1) a general adoption of DCR in US MTFs from 2006 onwards. (2) Those who died during DCR period had more severe injuries and had an increased likelihood of severe head injury. (3) A decrease in deaths among potentially salvageable patients post DCR adoption.

Conclusions 1 and 2 are clearly demonstrated in the data presented and it is sensible to reach these conclusions. The third conclusion the authors draw is, however, unsupported by the data presented. The core data in this paper does show that in order to have died after general adoption of DCR one had to have more severe injuries and a worse TRISS. Unfortunately, although one can conclude the aforementioned, it is impossible to confidently extrapolate and attribute this perceived survival benefit directly to DCR. The inability to do this is that throughout OIF and OEF significant developments/changes took place that could have equally contributed to the observed changes observed. These include but are not limited to improved pre-hospital care, decreased average casualty evacuation time, more established treatment facilities and the fact that the medical providers gained experience and increasing confidence/competency in dealing with the modern day battlefield injury. As this was an observational epidemiological study none of these factors were controlled, consequently strong conclusion regarding pre and post 2006 causation cannot be confidently reached.

4. Did results get omitted and why? No. All results that were highlighted in the initial search (admitted to MTF with recordable observations and subsequently died) were included for analysis.

5. Did they suggest areas of further research? Yes. The authors noted a trend of increasingly severe major head/brain injury and thus suggested research into prevention and optimal treatment of these.

6. Did they make recommendations based on the results and are they appropriate? No.

7. Is the study relevant to my clinical practice? Yes. Our hospital is major trauma center (Level 1 Trauma Center) and also the Role 4 hospital for the British Armed Forces, thus we are likely to treat patients with significant traumatic injury in need of DCR (or who have received DCR). A caveat to this would be that the military personnel treated in our center have survived their initial injury and have been transferred back to the UK, as a result of this DCR will either be ongoing or will have already occurred slightly altering the situational setting. A second limitation would be that trauma within the UK is unlikely to be due to significant blast injury or highvelocity penetrating injury as is the case in this case series.

8. What level of evidence does this study represent? 3 (Evidence from non-experimental descriptive studies, such as comparative studies, correlation studies, and case-control studies).

9. What grade of recommendation can I make on the results alone? D (directly based on Level 4 evidence or extrapolated recommendations from Level 1, 2 or 3 evidence).

10. What grade of recommendation can I make when this study is considered along with other available 
evidence? C. Although the concept of DCR is widely accepted there are as of yet no true randomized or even controlled trials. Data are retrospective and predominantly observational. Despite this, anecdotally the outcomes appear favorable and thus a 'major transfusion protocol' which attempts to provide a 1:1:1 with potential addition of adjunct factors seems advisable.

11. Should I change my practice because of these results? No. The current practice in our center is guided by our 'massive transfusion protocol'. This consists of a first pack consisting of $4 \times$ PRBC and $4 \times$ FFP providing 1:1 resuscitation. After this all subsequent packs released contain $4 \times$ PRBC, $4 \times$ FFP and $1 \times$ adult therapeutic dose (ATD) platelets; this provides a ratio of $1: 1: 1$. In light of our current standard practice, which is in essence DCR, we do not need to change our practice. Even if this was not our standard practice on the results of this paper alone one should not change their practice. The reason is that this study when taken on its own has many potential confounding factors thus limiting its application and power to change practice.

12. Should I audit my current practice because of these results? No.

\section{Disclaimer}

The opinions and/or assertions in this article are the personal opinions of the authors. They are not to be construed as official or as reflecting the views of the Army Medical Services, British Armed Forces, Ministry of Defence or University Hospital Birmingham Trust.

\section{References}

1. Hess JR, Holcomb JB, and Hoyt DB. Damage control resuscitation: the need for specific blood products to treat the coagulopathy of trauma. Transfusion 2006; 46: 685-686.

2. Holcomb JB. Damage control resuscitation. J Trauma 2007; 62(6 suppl): S36-S37.

\section{Appraised by:}

Charles Handford and Tomasz Torlinski, Critical Care Unit The New Queen Elizabeth Hospital Birmingham Mindelsohn Way Edgbaston Birmingham B15 2WB 